\title{
Wandel des Völkerrechts - Herausforderungen an die Steuerungsfähigkeit des Rechts im Zeitalter der Globalisierung ${ }^{1}$
}

\section{Verdichtung der internationalen/transnationalen Beziehungen - Verdichtung der Rechtsordnung - Verrechtlichung}

\section{Einführung}

Der Adolf Arndt-Kreis ist einer auf das Ganze gerichteten Betrachtung verpflichtet. Deshalb ist die Einführung, die in diesem Beitrag versucht wird, nicht allein auf die Probleme gerichtet, die Gegenstand des Tagungsprojekts sind (Hegung von Gewalt und Menschenrechte). Es soll vielmehr versucht werden, diese Probleme in einer Gesamtbetrachtung der Entwicklung des Völkerrechts einzuordnen. So dürften sich Zusammenhänge erschließen, die auch für das Tagungsprojekt eine Rolle spielen.

Die transnationalen Beziehungen haben sich in den letzten Jahrzehnten in dramatischer Weise intensiviert und verdichtet (Globalisierung). Das ist vielfach konstatiert worden und hat vielfältige Gründe. Politische, gesellschaftliche und technische Entwicklungen haben zu einer vorher nicht gekannten Freizügigkeit für Menschen, für wirtschaftliche und geistige Leistungen - und für Probleme geführt. Das Ergebnis ist internationale Verflechtung, Interdependenz. Diese Intensivierung der internationalen Beziehungen hat durch die Umbrüche des Jahres 1990 einen kräftigen Schub erhalten. Die Implosion des Sovietreiches bedeutete mehr als den Wegfall eines militärischen Gegengewichts gegen die von den USA geführte Allianz. Sie bedeutete das Ende der Abschottung eines wesentlichen Teils des Staatensystems. Die sozialistische Planwirtschaft als Alternative zu einem freiheitlichen Wirtschaftssystem war mit dem Sovietreich verschwunden. Das bedeutete auch einen Paradigmenwechsel in den internationalen Wirtschaftsbeziehungen. Ohne diesen Paradigmenwechsel wäre wohl die zeitgleich damit geschehende Gründung der WTO in der Uruguay-Runde nicht möglich gewesen. Die Freizügigkeit und Interdependenz weltweit, die das Zeitalter der Globalisierung kennzeichnen, wären ohne das Ende des Ost-West-Konflikts so nicht denkbar, sind aber nicht allein dadurch bedingt.

Freizügigkeit und Interdependenz sind ein politisches, gesellschaftliches und wirtschaftliches Phänomen. Der Staat verschwindet nicht, aber seine Grenzen werden durchlässiger. Träger dieser Intensivierung sind darum nicht nur oder nicht einmal hauptsächlich die Staaten, sondern auch nicht-staatliche Akteure unterschiedlicher Art (wirtschaftliche Akteure, transnationale soziale und politische Interessengruppen). Das System wird auch durch nicht-staatliche Akteure in Frage gestellt (transnationales Verbrechen, Terrorismus).

Mit dieser Intensivierung und diesem Wandel der transnationalen Beziehungen wachsen und ändern sich die Regelungsaufgaben der internationalen Rechtsordnung.

1 Initiativreferat für ein Projekt des Adolf-Arndt-Kreises. 
Globalisierung ist eine Herausforderung an die Steuerungsfunktion des Rechts. Sie führt dazu, dass wesentliche Ordnungsaufgaben des Rechts nicht mehr allein auf staatlicher Ebene erfüllt werden können, sondern dass es stärker als je zuvor internationaler Regelung bedarf. Ob und inwieweit das Völkerrecht diesen Anforderungen gerecht wird, ist entscheidend. In diesem Beitrag soll der Frage nachgegangen werden, in welcher Weise sich das Völkerrecht gewandelt hat, um diesen Herausforderungen gerecht zu werden, und ob es ihnen auch wirklich gerecht wird.

Es ist nicht zu leugnen, dass die Intensivierung der transnationalen Beziehungen zu einer Verdichtung der Rechtsbeziehungen geführt hat. Diese Verdichtung wird als »Verrechtlichung«, »legalization« bezeichnet. Die Entwicklung des Rechts folgt einer Entwicklung des politisch-sozialen Umfeldes. Allerdings ist diese Entwicklung nicht selbstverständlich oder automatisch. Es ist ja durchaus festzustellen, dass die Akzeptanz und Sinnhaftigkeit völkerrechtlicher Bindung immer wieder infrage gestellt wird. Dabei ist allerdings genauer hinzusehen, ob ein Widerstand der rechtlichen Bindung, der rechtlichen Steuerung überhaupt oder nur einem bestimmten rechtlichen Inhalt gilt.

Diese Verdichtung der internationalen Rechtsordnung lässt sich in unterschiedlichen Bereichen zeigen.

\section{Internationale Wirtschaftsbeziehungen}

Im Bereich der Wirtschaftsordnung ist vor allem zu nennen das Vertragssystem der WTO mit ihren eigenen Streitregelungsverfahren. Es ist ein komplexes Regelwerk der Sicherung freien Handels mit Waren und Dienstleistungen entstanden, das einem marktwirtschaftlichen Credo folgt.

Daneben ist aber auch eine Fortentwicklung der Institutionen des internationalen Finanzsystems (ein notwendiges Gegenstück zum System des Handels mit Waren und Dienstleistungen) festzustellen. Dieses System hat zwei große Krisen durch eine sehr flexible Fortentwicklung des rechtlichen Instrumentariums gemeistert. Die erste Krise war der Zusammenbruch des Gold-Dollar-Standards des Bretton Woods-Systems nach 1970, wobei die Anpassung der Satzung des IWF, die nunmehr ein System flexibler Währungskurse ermöglicht, nur bereits außerhalb des Rechts erfolgte Entwicklungen nachzeichnen konnte. Die zweite ist die Verschuldungskrise seit 1980, in der die Institutionen rechtstechnisch das Liquiditätsproblem relativ rasch beherrschten, ohne freilich das der Krise zugrunde liegende wirtschaftliche Ungleichgewicht wirklich anzugehen, jedenfalls zunächst.

Nicht in allen Problemen führt die Verdichtung der internationalen Beziehungen zu neuen Entwicklungen von Rechtsnormen. So sollte es auf einem globalen Markt auch globale Regeln gegen Wettbewerbsbeschränkungen geben. Es gibt sie aber nicht. Der Regelungsbedarf wird vielmehr durch eine informelle Abstimmung zwischen den drei wesentlichen »nationalen« Regulierungsbehörden (Brüssel, Washington, Tokyo) befriedigt.

Das Problem nicht-staatlicher Akteure wird im internationalen Wirtschaftsrecht seit den späten 60er Jahren vor allem im Hinblick auf große weltweit agierende Wirt- 
schaftsunternehmen (transnational corporations) diskutiert. Große Unternehmen begegnen den Staaten vielfach auf Augenhöhe. Gerade in Bezug auf diese Unternehmen haben die Staaten aber ihren Exklusivitätsanspruch als Völkerrechtssubjekte nicht aufgegeben. Es wurden andere Wege gefunden, mit Regeln, die nicht Völkerrecht sind, sog. codes of conduct, Unternehmen unmittelbar zu Adressaten eines internationalen Regelwerks zu machen.

Trotz der bemerkenswerten Entwicklung der internationalen rechtlichen Ordnung der Wirtschaft ist eine grundlegende Herausforderung an diese Ordnung nicht bewältigt: nämlich das unakzeptable Wohlstandsgefälle. Dass vor dieser Herausforderung erst die Politik und ihr folgend das Recht versagt haben, deutet auf ein grundlegendes Defizit der internationalen Ordnung hin, auf das zurückzukommen ist.

\section{Internationale Sozialordnung}

Mit der letzteren Frage stellt sich auch die Frage nach einer internationalen Sozialordnung. Das Problem wurde schon am Ende des Ersten Weltkrieges gesehen, als mit dem Völkerbund die Internationale Arbeitsorganisation (ILO) geschaffen wurde. Sie hat ein eindrucksvolles System arbeits- und sozialrechtlicher Verträge geschaffen. Gerade im Bereich der sozialen Sicherheit ist aber der Staat der unverzichtbare Akteur geblieben. Finanziell leistungsfähige Systeme der sozialen Sicherheit gibt es nur im Staat, selbst innerhalb der EU.

Bei der Einbindung nicht-staatlicher Akteure ist die ILO insofern vorbildlich, als in ihr schon seit ihrer Gründung das Plenarorgan nicht den Vertretern der Mitgliedstaaten vorbehalten ist. Vielmehr sind in ihm die Vertreter der Arbeitnehmer- und Arbeitgeberorganisationen gleichberechtigt vertreten. Beide Arten von Koalitionen haben auch sonst in der ILO erhebliche Verfahrensrechte.

\section{Schutz der Umwelt}

Im internationalen Umweltschutz entwickeln sich seit den 70er Jahren Vertragssysteme. Auf sie im einzelnen einzugehen, würde den Rahmen dieses Beitrags sprengen.

In den 90er Jahren wird ein Herzstïck dieser Entwicklung der Klimaschutz. Mit dem Kyoto-Protokoll wird in großer Kühnheit ein hoch komplexes, in wesentlichen Teilen international administriertes Regelungssystem geschaffen, das ohne Vorbild ist, aber vorbildhaft werden könnte.

$\mathrm{Ob}$ dieses System so belastbar ist, dass es die rechtlich vorgegebenen Ziele auch wirklich erfüllt, bleibt allerdings abzuwarten. Der Regelungsansatz des Kyoto-Protokolls, ein quantitatives Reduktionsziel für jeden einzelnen Staat festzulegen, ergänzt durch sog. flexible Mechanismen, ist ja nicht unumstritten. Deswegen ist auch nicht ausgemacht, ob er das Jahr 2012 überlebt. Das Abschlusspapier der Bali-Konferenz hat das jedenfalls noch nicht entschieden.

Nicht-staatliche Akteure sind an der Entwicklung und Durchsetzung des Umweltvölkerrechts in vielfältiger Weise beteiligt. Ein besonderes Markenzeichen des Kyoto-Protokolls ist die Einbindung von Wirtschaftsunternehmen im Emissionshandel. Praktisch ist der nicht-staatliche Bereich auch in den anderen flexiblen Mechanismen involviert. 
Ob und inwieweit die Explosion umweltrechtlicher Regeln, die das Völkerrecht der letzten Jahrzehnte des 20. Jahrhunderts kennzeichnet, die anstehenden Probleme der Erhaltung der natürlichen Umwelt wirklich wirksam kontrolliert, ist noch nicht endgültig zu sagen. Deswegen ist das weitere Nachdenken über die Instrumente des Umweltrechts auf nationaler, regionaler und weltweiter Ebene erforderlich. Gerade in diesem Zusammenhang spielen nicht-staatliche Akteure eine erhebliche Rolle.

\section{Menschenrechte}

Die Entwicklung eines völkerrechtlichen Menschenrechtsschutzes ist wohl die zentrale Innovation des universalen Völkerrechts nach dem 2. Weltkrieg. Allerdings kam nach der Allgemeinen Erklärung der Menschenrechte die vertragsrechtliche Regelung des Menschenrechtsschutzes (mit Ausnahme von Europa) zunächst nur schleppend voran. Die beiden großen universalen Verträge, die sog. Pakte, 1966 in der Generalversammlung der VN angenommen, traten erst 10 Jahre später in Kraft. Seitdem haben sich allerdings die Vertragsgemeinschaften des Menschenrechtsschutzes und ihre Institutionen erheblich entwickelt - nicht immer ohne Kontroversen (Menschenrechtskommission, Menschenrechtsrat).

Große Fortschritte wurden vor allem in Europa durch die Osterweiterung der EMRK nach 1990 und die Vereinfachung und Ausdehnung des individuellen Gerichtsschutzes durch das 11. Zusatzprotokoll zur EMRK von 1994 erzielt. Erst durch dieses Zusatzprotokoll wird der EGMR seit 1997 für das Individuum unmittelbar zugänglich.

Dass durch diese eindrucksvolle Entwicklung des Vertragsrechts ein flächendeckender Menschenrechtsschutz erreicht ist, der auch Problemfälle erfasst, lässt sich so allgemein nicht sagen. Lücken der vertragsrechtlichen Bindungen und unterschiedliche Interpretationen, professionell vertretbare oder unvertretbare, lassen nur zu häufig unakzeptables menschenrechtswidriges Verhalten ungeahndet. Es stellt sich auch die Frage, wieweit das nationale Strafrecht immer seiner Aufgabe gerecht wird, menschenrechtswidriges Verhalten nicht-staatlicher Akteure zu verhindern und ob insoweit völkerrechtlich nachgebessert werden kann.

\section{Rechtliche Hegung von Gewalt}

Zur rechtlichen Hegung von Gewalt gibt es zwei Rechtsmassen, nämlich einmal die Normen, nach denen zu beurteilen ist, ob überhaupt militärische Gewalt eingesetzt werden darf (fälschlich ius ad bellum genannt, richtiger ist ius contra bellum), zum andern Normen über die Art und Weise der Gewaltanwendung (ius in bello), die Anwendung finden, wenn Gewalt nun doch, rechtswidrig oder rechtmäßig, angewandt wird.

Zentrale Norm der rechtlichen Hegung von Gewalt ist das allgemeine Gewaltverbot, in der Satzung der Vereinten Nationen und im Gewohnheitsrecht verankert. Praktische und rechtliche Hegung von militärischer Gewalt ist Kernaufgabe der Vereinten Nationen. In dieser Frage fällt heute zunächst ins Auge die neue Arbeitsfähigkeit des Sicherheitsrates, d.h. die Wiederbelebung des Kernstücks des Systems der Friedenssicherung, das die UN Charta 1945 errichtete. Diese Wiederbelebung war aber im Ergebnis nicht so erfolgreich, wie man sich das 1990 erhofft hatte. Die Welt ist seit- 
dem nicht friedlicher, sondern eher unfriedlicher geworden. Festzustellen ist jedoch ein deutlicher Wandel der Sicherheitsproblematik (Stichwort: neue Bedrohungen). Er hat auf der einen Seite zu erheblichen Unsicherheiten bezüglich des Normbestandes der völkerrechtlichen Friedenssicherung geführt (Ausdehnung der völkerrechtlichen Rechtfertigung der Anwendung von militärischer Gewalt, pre-emptive self-defence). Allerdings wird diese Ausdehnung des Selbstverteidigungsrechts nicht in der Form vertreten, dass eine rechtliche Bindung abgelehnt wird. Sie ist vielmehr in einen Rechtsdiskurs eingebunden. Andrerseits hat die Erkenntnis neuer Bedrohungen eine Intensivierung internationaler Kooperation hervorgerufen (Stichwort: Terrorismusbekämpfung).

Die zweite Ebene der rechtlichen Hegung von Gewalt ist das Recht der Regelung bewaffneter Konflikte. Es beruht auf einem alten Normenbestand, hat sich aber aufgrund der Erfahrungen von bewaffneten Konflikten immer wieder fortentwickelt. Die in den 70er Jahren begonnene Weiterentwicklung des Vertragsrechts (Zusatzprotokolle zu den Genfer Abkommen 1977) hat sich in den 80er Jahren fortgesetzt mit der UN-Waffenkonvention (einer Rahmenkonvention mit einem immer noch wachsenden System von Zusatzprotokollen), in den 90er Jahren dann mit Schritten vom Einsatzverbot für Waffen zur Rüstungskontrolle (Chemiewaffen, Landminen), wozu sich seit neuestem ein Vertrag über Streumunition gesellt. Diese Verbindung von Rüstungskontrolle und Einsatzverboten ist jedenfalls eine wesentliche Neuentwicklung.

Ein wichtiger Schritt zur besseren Durchsetzung des Rechts bewaffneter Konflikte ist die Entwicklung der Internationalen Strafgerichtsbarkeit, zunächst mit den Sondergerichtshöfen für Jugoslawien und Ruanda, dann 1998 mit dem Statut eines Internationalen Strafgerichtshofes. Dessen Zuständigkeit umfasst eigentlich auch das ius contra bellum. Allerdings kann die einschlägige Bestimmung über das Verbot der Aggression erst in Kraft treten, wenn im Wege der Vertragsänderung eine Definition der Aggression hinzugefügt wird. Obwohl zu diesem Zweck pflichtgemäß ein Ausschuss eingesetzt wurde, ist nicht davon auszugehen, dass eine solche Vertragsänderung in absehbarer Zeit zustande kommt.

Die neuen Bedrohungen mit geänderten Konfliktbildern (sog. neue Kriege) stellen auch eine Herausforderung für das Recht der Regelung bewaffneter Konflikte dar. Ein klassischer Mechanismus, die Einhaltung von Völkerrecht sicherzustellen, nämlich das Prinzip der Gegenseitigkeit, wird an sich rechtlich zurückgedrängt. Wenn eine Seite Gefangene foltert, rechtfertigt es nicht, dass die andere Seite Gleiches tut. Dennoch ist Gegenseitigkeit faktisch ein wichtiges Element für das Funktionieren des Rechts bewaffneter Konflikte geblieben, und deshalb entwickeln sich in asymmetrischen Konflikten erhebliche Anreize zum Rechtsbruch. Das Problem der Asymmetrien wird noch dadurch verstärkt, dass an bewaffneten Konflikten in unterschiedlicher Weise nicht-staatliche Akteure beteiligt sind. Der klassische juristische Ansatz des Umgangs mit neuartigen Problemen ist die vorsichtige Anwendung und Anpassung überkommener Prinzipien. Das ist gerade in dem Fragenkreis der Hegung von Gewalt nicht spannungsfrei verlaufen. Es sind andererseits neue rechtliche Instrumente entwickelt worden, etwa »gezielte Sanktionen « des Sicherheitsrats gegen Helfer des Terrorismus. 
Behauptungen, das Völkerrecht der Hegung von Gewalt sei in wesentlichen Teilen obsolet geworden, treffen nicht zu. Es bleibt bei dem Versuch, den neuen Herausforderungen in einem Rechtsdiskurs zu begegnen. Das Phänomen der Verrechtlichung hat die rechtliche Hegung von Gewalt nicht ausgespart. Allerdings will es trotz dieser positiven Entwicklungen oft erscheinen, als sei gerade der Versuch der rechtlichen Hegung der Gewalt ein Kampf gegen die Hydra, bei der das Völkerrecht noch nicht zur Form eines Herkules aufgelaufen ist.

\section{Die Fragmentierung der transnationalen Rechtsordnung.}

Träger der Verdichtung der Rechtsbeziehungen sind spezifische Institutionen, die jeweils eigene Regelungsregime hervorbringen. Diese Regime führen ein (oft sehr dynamisches) Eigenleben.

Dies führt erstens zu einer Fragmentierung der Rechtsbildung. Ein charakteristisches Phänomen sind Vertragsgemeinschaften, die immer komplexere Regelungssysteme hervorbringen (UNEP, UNFCCC, Kyoto-Protocol, Marrakech Accords): Während bestehende Organisationen »Geburtshilfe« leisten, bilden sich Vertragsgemeinschaften, deren Entwicklung durch eigene Netzwerke (vernetzte Bürokratien und Interessengruppen) getragen wird. Die Beteiligung nicht-staatlicher Akteure in diesen Netzwerken ist ganz erheblich. Ohne die Eigendynamik dieser Regelungsregime wäre es gar nicht möglich, den Regelungsbedarf der internationalen Ordnung zu stillen.

Dieses Phänomen führt zweitens zu einer Fragmentierung der Rechtsdurchsetzung: Es zeigt sich eine Proliferation internationaler Streitregelungsmechanismen und Rechtsprechungsorgane. Genauer betrachtet ergibt sich folgendes Bild:

- nicht regime-spezifische Streitregelungsmechanismen (Internationaler Gerichtshof, Haager Schiedshof);

- regimespezifische Kontrollmechanismen (z.B. die sehr wichtigen Berichtssysteme mit Überprüfungsmechanismen);

- regimespezifische Streitregelungs- und insbesondere Gerichtsinstitutionen (Seerechtsgerichtshof, WTO-Streitregelungsmechanismus, Gerichte oder Kommissionen nach Maßgabe der verschiedenen Menschenrechtsverträge, internationale Strafgerichtshöfe).

In dem Gesamt-»System « der Rechtsdurchsetzung wird auch die Rolle nationaler Rechtsprechungsorgane gestärkt. In dem (sogleich zu behandelnden) MehrebenenSystem ist das Völkerrecht auf nationale Rechtsdurchsetzung angewiesen, die auch in unterschiedlicher Weise geleistet wird (Stichwort: »complementarity« gemäß dem Statut des IStGH). Dabei leistet nationale Zuständigkeit aufgrund des Weltrechtsprinzips einen wesentlichen, allerdings oft streitbefangenen Beitrag. Diese Bedeutung der nationalen Rechtsdurchsetzung trägt allerdings weiter zur Fragmentierung bei, da sie die Einheitlichkeit der Auslegung bestimmter Rechtsregime infrage stellt.

Angesichts dieses Befundes der Fragmentierung stellt sich die Frage der Einheit der Rechtsordnung. Dabei handelt es sich nicht nur um ein zu bestreitendes theoretisches Postulat, sondern auch um ein ganz praktisches Problem, da die Fragmentierung dazu 
führt, dass Rechtsunterworfene aus unterschiedlichen Rechtsregimen inkompatible Handlungsanweisungen erhalten können. Die Lösung dieser Frage ist noch offen. Es fehlt nicht an punktuellen Versuchen, einzelnen Widersprüchlichkeiten abzuhelfen (Stichwort: Handel und Umwelt). Eine überwölbende Institution, die einen Ausgleich zwischen unterschiedlichen Regimen umfassend und systematisch bewirken könnte, gibt es jedoch (noch?) nicht.

\section{Wertorientierung und das Problem der Konstitutionalisierung}

Eine in dieser Weise intensivierte Rechtsordnung ist mehr als die rein rechtstechnische Regelung eines Geflechts von Beziehungen. Sie bedarf einer besonderen Legitimation, sie ist nicht vorstellbar ohne eine Orientierung an Gemeinwohlvorstellungen. Keine Rechtsordnung kann letztlich ihre Ordnungsaufgabe ohne Akzeptanz der Rechtsunterworfenen erfüllen. Das bedeutet aber in der gewandelten internationalen Ordnung, wie sie kurz skizziert wurde, nicht nur Akzeptanz durch die »Staaten«, d.h. die staatlichen Bürokratien, sondern durch ein viel weiteres Netzwerk nicht-staatlicher Akteure. Stichworte sind Frieden und Schutz vor Gewalt, Wohlstand, Verteilungsgerechtigkeit, Schutz der Umwelt, Sicherung der Interessen zukünftiger Generationen.

Der Schutz des Gemeinwohls ist eine traditionelle staatliche Aufgabe. Übernimmt die internationale Ordnung Teile dieser Aufgabe, so stellt sich die Frage, ob sie eine $»$ Verfassung « besitzt, die diesen Namen verdient (Debatte um »Konstitutionalisierung $\ll)$.

Bei aller semantischen Verwirrung ist als Kern wohl festzuhalten: Konstitutionalisierung bedeutet die Verpflichtung einer rechtlichen Ordnung auf Grundwerte und Regeln über politische Entscheidungsprozesse. Die heutige internationale Ordnung besitzt beides, wenn auch in unvollkommener Form.

Verschwindet deshalb die staatliche Souveränität in einer Verfassung der Staatengemeinschaft? Nein, aber sie wandelt sich. Diesen Befund reflektiert das von Kofi Annan geprägte Schlagwort »Redefining sovereignty«. Souveränität wird oft eingesetzt als Schlagwort für die alleinige Ausrichtung staatlichen Verhaltens an oft kurzfristig verstandenen staatlichen Eigeninteressen. Sie bleibt ein zentraler Faktor in der Realität der internationalen Beziehungen. Eine Durchsetzung von Gemeinnutz gegen Eigennutz bleibt eine bislang unerfüllte Aufgabe der internationalen Ordnung.

\section{Die räumliche Dimension der Verdichtung und Fragmentierung - regionale Regime}

Die verschiedenen Regionen der Welt haben eigene Institutionen und eigene Vertragsgemeinschaften hervorgebracht, allerdings aufgrund unterschiedlicher politischer, sozialer und wirtschaftlicher Strukturen sowie aufgrund unterschiedlicher politischer Geographie in unterschiedlicher Weise. Schon die Bildung von Regionen und Subregionen der Integration ist variantenreich. Politische und wirtschaftliche Organisationen spielen eine unterschiedliche Rolle. So intensiv wie in Europa ist die regionale Integration freilich sonst auf der Welt nicht. 
In der westlichen Hemisphäre bildeten sich schon im 19. Jahrhundert Institutionen der Zusammenarbeit. Die politische Integration der »Americas « steht unter einer starken Nord-Süd-Spannung. Für die subregionale Integration mit wirtschaftlichem Schwerpunkt bedeuten NAFTA und Mercosur Fortschritte. Afrika weist mit der AU als Nachfolgerin der OAU eine sich entwickelnde Institution politischer Zusammenarbeit und Friedenssicherung auf. Die subregionale Integration ist in Ostafrika früh gescheitert, heute spielt sie in Westafrika und im südlichen Afrika eine erhebliche Rolle. Die Liga der Arabischen Staaten hat mit enormen inneren und äußeren Schwierigkeiten zu kämpfen, ist aber dennoch ein bedeutender Faktor der innerarabischen Konfliktlösung und der Abstimmung arabischer Politik nach außen, nicht zuletzt gegen Israel. In Asien ist es vor allem die subregionale Organisation der ASEAN, die als ein Forum der Konfliktbearbeitung durchaus erfolgreich ist, obwohl ihre Institutionalisierung immer noch relativ schwach ist. Eine regional weiter greifende ostasiatische Integration scheitert an dem immer noch problematischen Verhältnis zwischen Japan und China einerseits, zwischen diesen Staaten und dem Rest der Region andererseits.

Auch auf regionaler Ebene gibt es das Phänomen und Problem der Fragmentierung. Allein in Europa können wir vier unterschiedliche nebeneinander, oft miteinander, manchmal auch gegeneinander agierende Institutionen feststellen: die Europäischen Gemeinschaften/EU, der Europarat mit einer Fülle von Vertragsgemeinschaften, die OSZE, die NATO/WEU. Das Verhältnis dieser Institutionen zueinander und zu den Vereinten Nationen etwa bei der Bewältigung der Balkankrise war zunächst Gegenstand von Improvisation, nunmehr wird es zum Gegenstand politikwissenschaftlicher und juristischer Forschung.

Insgesamt ist das Phänomen der Regionalisierung sehr unübersichtlich - aber ohne diese Regionalisierung ist die Lösung der Steuerungsaufgaben der internationalen Ordnung heute nicht vorstellbar.

\section{Vertikale Verdichtung: rechtliche Ordnung im Mehrebenensystem}

Eine sachlich angemessene Steuerung der transnationalen Lebenssachverhalte ist nicht möglich ohne eine Verteilung der Steuerungsfunktionen auf verschiedene Ebenen der öffentlichen Gewalt. Das lässt sich in verschiedenen Regelungsregimen in unterschiedlicher Weise zeigen. Im Klimaschutz gibt die völkerrechtliche Ebene (das Kyoto-Protokoll) Gesamt-Ziele der Reduktion von Emissionen vor. Diese werden in der EU zunächst konkretisiert und umgesetzt, dann weiter konkretisiert auf der Ebene der Mitgliedstaaten. Das endet auf der kommunalen Ebene etwa bei der Frage einer Bebauungsplanung, die Erfordernisse des Klimaschutzes einbeziehen muss. Die Steuerungsfunktion von Regelungsregimen muss schließlich immer menschliches Verhalten erfassen. Dies geschieht in aller Regel auf unteren Ebenen der Regierungsgewalt.

Die sinnvolle Ausgestaltung einer rechtlichen Steuerung im Mehrebenensystem ist eine zentrale Frage des modernen internationalen Rechts. 


\section{Wandel des Völkerrechts: vom Völkerrecht für Staaten zum Völkerrecht für Men- schen?}

Traditionell wird das Völkerrecht als die Rechtsordnung definiert, die die Beziehungen zwischen Staaten regelt. Der Einzelmensch war nicht Adressat dieser Rechtsordnung. Er wurde durch sie nur faktisch, nicht rechtlich begünstigt oder belastet.

Nach extremen Ereignissen der Menschenverachtung hat das Völkerrecht in der zweiten Hälfte des 20. Jahrhunderts einen differenzierten völkerrechtlichen Menschenrechtsschutz entwickelt, ein tief greifender Paradigmenwechsel des Völkerrechts. Grundgedanke dabei war zunächst der Schutz des Individuums gegen den Staat, dessen Hoheitsgewalt es unterworfen ist. Als Form der Verwirklichung dieses Menschenrechtsschutzes steht zunächst der individuelle Rechtsschutz im Vordergrund, der im Mehrebenensystem unterschiedliche Ausgestaltungen erfährt (Recht auf nationalen Rechtsschutz, unmittelbare Beschwerde des Individuums an internationale Instanzen des Menschenrechtsschutzes).

Dem sind gerade in den letzten zwei Jahrzehnten weitere Dimensionen des Menschenrechtsschutzes hinzugetreten. Zum ersten wurde deutlich, dass es nicht nur Staaten, sondern auch internationale Institutionen sind, die durch ihre Hoheitsakte unmittelbar in eine zu schützende Rechtssphäre des Individuums eingreifen. Daraus hat der EuGH seit Beginn der 70er Jahre, und dann die EG/EU insgesamt die Konsequenz gezogen und einen Grundrechtsschutz des Individuums gegen die Gemeinschaft entwickelt. Gegenwärtig stellt sich diese Frage für den Sicherheitsrat der Vereinten Nationen, wo ähnliche Entwicklungen noch auf erhebliche Widerstände treffen.

Des Weiteren rückt die Verpflichtung der internationalen Gemeinschaft in den Blick, positive Maßnahmen zum Schutz der Menschenrechte zu treffen. Im Pakt über wirtschaftliche, soziale und kulturelle Rechte wird schon gesagt, dass die Ermöglichung dieser Rechte nicht nur die Aufgabe jedes einzelnen Staates für sein Staatsgebiet ist, sondern dass zur Erfüllung dieser Aufgabe eine Pflicht zur Zusammenarbeit besteht. Daran schließt sich die Debatte um Schutzpflichten bei schweren Menschenrechtsverletzungen (responsibility to protect) an. Umfang und Inhalt solcher Schutzpflichten sind noch unklar. Jedenfalls hat sich die Idee, dass solche Schutzpflichten einseitige militärische Aktionen selbsternannter Schützerstaaten (sog. humanitäre Intervention im Kosovo) rechtfertigen könnten, nicht durchgesetzt. Um so wichtiger ist die entsprechende Verantwortung des Sicherheitsrats der Vereinten Nationen.

Ein drittes Problem ist die Bedeutung des Menschenrechtsschutzes für andere Bereiche des Völkerrechts. Das Problem ist eine Folge der Fragmentierung: Inwieweit haben die Regeln des Menschenrechtsschutzes, die sich in ihren eigenen Regelungsregimen entwickelt haben, Wirkungen in anderen Rechtsregimen? Stichworte sind etwa der Schutz des Menschen in der internationalen Wirtschaftsordnung, Recht auf eine gesunde Umwelt, insbesondere (hochaktuell) ein Recht auf Wasser, und die parallele Anwendung der Menschenrechte und des Rechts bewaffneter Konflikte.

Das Problem hat auch wichtige prozedurale Konsequenzen. Wo immer es gelingt, eine Frage anderer Regelungsregime als Menschenrechtsfrage zu formulieren, übernimmt der prozedurale Menschenrechtsschutz gleichzeitig die Funktion der Durchset- 
zung anderer Regelungsregime. Die aus der Sicht des Individuums objektive Ordnung der zwischenstaatlichen Beziehungen erhält so eine subjektive Komponente (Beispiele: Durchsetzung des Rechts bewaffneter Konflikte vor Gerichten im Wege des individuellen Rechtsschutzes [Fälle Distomo und Vavarin], Widerstand gegen die Wiederaufnahme französischer Atomversuche 1996 durch Rechtsbehelfe des Menschenrechtsschutzes). Die Parallelität von Menschenrechten und humanitärem Recht spielt für das ius in bello praktisch eine sehr wichtige Rolle. Sie für das ius contra bellum fruchtbar zu machen, ist angedacht, aber (noch?) nicht sehr erfolgreich.

Die Entwicklung des Menschenrechtsschutzes ist aber nicht die einzige treibende Kraft für einen Wandel des Völkerrechts. Nichtstaatliche Akteure spielen als Normadressaten und als Beteiligte am Normsetzungs- und am Normdurchsetzungsprozess eine immer größere Rolle. Bislang rein zwischenstaatliche Verfahren öffnen sich für nichtstaatliche Akteure. Völkerrecht wird heute nicht mehr nur von staatlichen Bürokratien für staatliche Bürokratien gemacht, sondern von einer Vielfalt von Akteuren für staatliche Bürokratien, für soziale Gruppen, für den Menschen.

\section{Wandel des Völkerrechts: die »Pole« der internationalen Beziehungen}

Trotz des beschriebenen Wandels bleibt die Machtstruktur im Staatensystem eine wesentliche Determinante des internationalen Rechts. Das Recht entwickelt sich (oder es entwickelt sich gerade nicht) in politischen Kräftefeldern. So war die internationale Ordnung in den ersten vier Jahrzehnten nach dem zweiten Weltkrieg stark geprägt durch die bipolare Struktur des weltpolitischen Kräftefeldes. Das Funktionieren oder genauer Nichtfunktionieren der Vereinten Nationen war dadurch bedingt (sog. automatisches Veto).

Dies hat sich durch die Implosion des Sowjetreiches 1990 nachhaltig geändert. Das automatische Veto gibt es so nicht mehr. Dies hat zu einer erneuten Handlungsfähigkeit des Sicherheitsrats geführt.

In der Zeit nach 1990 wurde vielfach erörtert, ob sich die internationale Ordnung von einem bi-polaren zu einem unipolaren System geändert habe, da nur eine Supermacht verblieben sei. In der Tat gibt es Elemente der amerikanischen Außenpolitik, die von einem solchen Konzept inspiriert sind (Stichwort»Unilateralismus«). Bei genauerem Hinsehen kann das jedoch nicht, jedenfalls nicht ohne Einschränkung gelten.

Auf der einen Seite ist der Sicherheitsrat ein zentrales Element der internationalen Friedensordnung geworden. Die Vereinigten Staaten können im Sicherheitsrat zwar Alles verhindern, aber nicht Alles positiv bewirken (Beispiel Veto-Praxis insbesondere in Bezug auf den Nahost-Konflikt). Die Situation hat zu einer natürlichen Allianz der Staaten geführt, die Alles verhindern können (P5, die »Oligarchen«; Beispiel IranSanktionen). Selbst angesichts der Tatsache, dass die P5 die einzigen »offiziellen« Atommächte sind, spiegelt diese Entscheidungssituation weniger die Machtsituation als die Perpetuierung eines historischen Privilegs, das zu erhalten gemeinsames Interesse der P5 ist. Deutschland, Japan, Indien und Brasilien sind gegenüber diesem Befund offenbar machtlos. 
Das traditionelle Modell einer multipolaren Ordnung war wohl geprägt durch Systeme wie das europäische Konzert des 19. Jahrhunderts, das sich in einem unstabilen Gleichgewicht befand. Das heutige System der internationalen Ordnung ist wesentlich komplexer. Es ist ein kompliziertes Netzwerk, in dem es Akteure (Staaten und nichtstaatliche Akteure) gibt, die in ihrer Bedeutung variieren.

\section{Plus ça change, plus c'est la même chose? Macht und Recht}

Die Struktur der internationalen Ordnung wirft also auch heute die alte Frage des Verhältnisses von Macht und Recht auf. Die uralte These der Völkerrechtskritik lautet, gerade in den internationalen Beziehungen gehe Macht vor Recht. Recht sei bedeutungslos. In der politischen Wissenschaft wird diese Schule als Realismus bezeichnet, was aber die Sache noch nie so ganz getroffen hat.

Es ist nicht zu bezweifeln, dass sich mächtige Staaten immer noch leichter über das Recht hinwegsetzen als weniger mächtige. Aber in der Netzwerkstruktur der internationalen Ordnung treibt das Recht die Kosten der Rechtswidrigkeit erheblich in die Höhe. Man kann es auch so formulieren: Die Verrechtlichung der internationalen Beziehungen relativiert die Bedeutung von Machtgefällen. Auch in Fragen der »high politics« spielt der Rechtsdiskurs eine erhebliche Rolle.

IX. Schlussfolgerungen: das Völkerrecht vor den Herausforderungen des 21. Jahrhunderts

Hat das Völkerrecht die gestiegenen und gewandelten Herausforderungen an seine Steuerungsfähigkeit im 21. Jahrhundert bewältigt? Eine differenzierte Antwort auf diese Frage muss zwischen positiven Entwicklungen und verbliebenen Problemen unterscheiden.

\section{Positive Entwicklungen}

Die Nachfrage nach rechtlichen Regelungen wird vor allem durch eine Dynamisierung von Rechtsbildungsprozessen in speziellen Regelungsregimes (universell oder regional) befriedigt. Dies ist ein wesentliches Element von Verrechtlichung.

Die Verfahren der Umsetzung und Durchsetzung des internationalen Rechts sind in hohem Maße intensiviert und diversifiziert.

Die Intensivierung der Rechtsfortbildung und -durchsetzung beruht nicht nur auf einer Schaffung neuer Institutionen, sondern auch auf einer Diversifizierung der Akteure. Organisationen der Zivilgesellschaft spielen in beiden Bereichen eine erhebliche Rolle, die die Rechtsbildung in Richtung auf das Gemeinwohl dynamisiert und die Rechtsdurchsetzung verschärft. Rule of law hat in der Tat in der internationalen Ordnung einen hohen Stellenwert erhalten.

Diese Entwicklung spart auch Fragen der hohen Politik nicht aus, auch und gerade bei der rechtlichen Hegung von Gewalt. Hier ist freilich das Gewicht der Macht gegenüber dem Recht am stärksten. 


\section{Probleme}

Die Dynamisierung der rechtlichen Entwicklung wird durch eine Fragmentierung erkauft, die zu Normwidersprüchen führen kann und auch geführt hat. Eine Institution, die umfassend solche Widersprüche ausräumen könnte, gibt es nicht.

Letzteres ist nur eines der Probleme, man kann auch von Defiziten sprechen, die sich aus dem Charakter des Völkerrechts als einer dezentralisierten Rechtordnung ergeben. Es bleibt vielfach bei einer Überbetonung staatlichen Eigeninteresses unter Berufung auf die staatliche Souveränität.

Die Bewältigung von Asymmetrien und die Einbindung vielfältiger nicht-staatlicher Akteure in das internationale Recht ist nur teilweise gelöst.

Die rechtliche Steuerung von internationalen Regelungsproblemen kann in der Regel nur auf mehreren Ebenen erfolgen. Der Durchgriff des Völkerrechts und internationaler Institutionen auf den einzelnen handelnden Menschen bleibt die Ausnahme. Dies stellt die Frage einer angemessenen Arbeitsteilung der Ebenen und der widerspruchsfreien Ausrichtung der Ebenen aufeinander.

Unterschiedliche Ausübung von Macht beeinträchtigt die Richtigkeitsgewähr für internationale Steuerung. Diese unterschiedlichen Machtfaktoren können bezeichnet werden als Mammon, Militär und Mehrheit. Wirtschaftliche und militärische Eigeninteressen von Staaten (ggf. auch von anderen relevanten Akteuren) stellen die Rechtsordnung immer wieder in Frage (Problem rechtswidriger Militärinterventionen). Eine ganz andere Machkonstellation sind die Mehrheitsverhältnisse in den universalen Organisationen, die gerade im Menschenrechtsbereich zu fragwürdigen Entscheidungen führen.

Das Konsensprinzip bleibt darum der entscheidende Faktor der Richtigkeitsgewähr für Maßnahmen der Steuerung internationaler Probleme. Dadurch wird die Entscheidung aber zum kleinsten gemeinsamen Nenner staatlicher Eigeninteressen, wie es von den entscheidenden Eliten, insbesondere den bürokratischen Eliten der Staaten definiert wird. Die Einbindung von Akteuren der Zivilgesellschaft in internationale Entscheidungsprozesse kann dieses Defizit mildern, aber nicht ausschließen. 